\title{
Prevent Cancer Foundation quantitative CT imaging workshop XVI: lung cancer, COPD and cardiovascular disease - on the cusp of transformation, Arlington, VA
}

\author{
James L. Mulshine ${ }^{1 *}$ D, Riccardo S. Avila, Daniel C. Sullivann ${ }^{2}$, David F. Yankelevitz ${ }^{4}$, Raúl San José Estépar ${ }^{5}$, \\ Laurie Fenton Ambrose ${ }^{6}$, Bruce Pyenson ${ }^{7}$ and Carolyn R. Aldigé ${ }^{8}$
}

\begin{abstract}
Throughout the course of this Workshop, members of the advocacy community, imaging researchers, computer and measurement scientists, clinicians and policy-focused workshop attendees engaged in cross-cutting discussions from innovative technical aspects of thoracic imaging to policy approaches to ensure equitable access to all at-risk individuals when implementing lung cancer screening services. A major aspect of these implementation discussions was how to efficiently collect routine thoracic CT-based screening with de-identified clinical outcomes data to support the development of robust imaging tools, including responsible Al development, to better detect and manage early lung cancer as well as other major tobacco-related thoracic diseases. A future vision involves routinely collecting a substantial fraction of every thoracic screening CT image to establish a large, curated collection of de-identified thoracic CT images with clinical outcome data to support open research for building better computational imaging tools for early thoracic disease management. Imaging researchers are positioned to develop much better workflow software tools to promote more efficient, outpatient management of the screening process for populations at-risk for lung cancer, especially with the rapid development of promising Al tools. Efficient and effective management tools for the large numbers of at-risk ever smokers could allow the primary care community to discuss lung cancer screening despite their heavy existing clinical demands. Supporting the primary care community in this fashion may significantly improve the current slow uptake of lung cancer screening and save many lives in the process.
\end{abstract}

Keywords: Lung cancer screening, Tobacco-related diseases, Quantitative imaging, Artificial intelligence, Image quality conformance measures, Image archive, HEDIS measures

\section{Introduction}

The Prevent Cancer Foundation (PCF) hosted the sixteenth annual Quantitative Imaging Workshop in Arlington, VA. The Workshop is a multi-disciplinary forum that seeks to advance quantitative CT imaging biomarkers for early thoracic disease management. The Workshop explores opportunities to use imaging to more reliably detect clinically aggressive early lung cancer and improve the

\footnotetext{
* Correspondence: jmulshin@rush.edu

${ }^{1}$ Rush University, Chicago, IL, USA

Full list of author information is available at the end of the article
}

management of the detected cancer. In particular, the Workshop supports the robust quantitative assessment of the growth of pulmonary nodules as emerging literature supports this is a sensitive and specific way to determine the aggressiveness of early lung cancers [1-4].

The Prevent Cancer Foundation has a long-standing interest in applying quantitative CT imaging to manage early lung cancer. When the first reports of the utility of spiral CT in detecting early lung cancer were published in 1999 by Dr. Claudia Henschke and her co-authors from Weill Cornell Medical Center

(c) The Author(s). 2020 Open Access This article is licensed under a Creative Commons Attribution 4.0 International License, which permits use, sharing, adaptation, distribution and reproduction in any medium or format, as long as you give

appropriate credit to the original author(s) and the source, provide a link to the Creative Commons licence, and indicate if changes were made. The images or other third party material in this article are included in the article's Creative Commons licence, unless indicated otherwise in a credit line to the material. If material is not included in the article's Creative Commons licence and your intended use is not permitted by statutory regulation or exceeds the permitted use, you will need to obtain permission directly from the copyright holder. To view a copy of this licence, visit http://creativecommons.org/licenses/by/4.0/. 
and Cornell University, there was considerable skepticism about the robustness of this report [1]. The Prevent Cancer Foundation took a proactive role in supporting the rapid evaluation of this screening approach. In 2000, in conjunction with the Roy Castle Foundation from the United Kingdom, and through the advocacy of Cherie Blair, wife of the Prime Minister of the United Kingdom, the Foundation convened the U.K.-U.S. Millennium Lung Cancer Workshop in the U.S. Executive Office Building to explore how a randomized CT trial could determine whether lung cancer screening (LCS) might really reduce deaths from lung cancer. At that workshop, Dr. Richard Klausner, then director of the National Cancer Institute (NCI), committed to conducting a definitive randomized trial to test whether CT screening could objectively reduce lung cancer-related mortality. When the next Director, Dr. Andrew C. von Eschenbach came to lead the NCI, the leadership of the Prevent Cancer Foundation, in collaboration with Professor Donald Coffey of Johns Hopkins University, strongly encouraged Dr. von Eschenbach to finalize the study design for the low dose screening trial and then to conduct this critical trial.

After an in-depth evaluation, Dr. von Eschenbach finalized and then launched the randomized screening trial that was called the National Lung Screening Trial (NLST) [5]. The NLST is the largest ever, single-site cancer screening trial and accrued over 53 thousand current or former smokers ahead of schedule. The trial conclusively demonstrated that LCS reduced lung cancer mortality by $20 \%$ [6]. This trial's landmark result of significant lung cancer-related mortality reduction ultimately led to the Center for Medicaid and Medicare's covering the cost of this new lung cancer screening service in the target populations across America [7, 8]. Reinforcing the importance of this finding, a recently published long-term (10 year) follow-up of the NLST subjects, confirmed the substantive impact of this screening approach and clarified that the observed rate of overdiagnosis was below 3\% [9]. This benefit has now been reproduced in a growing number of mature trial results from across the world, confirming this important cancer screening benefit [10-13].

Accordingly, in recognition of Dr. von Eschenbach's contribution to completing the NLST and providing gold-standard evidence of LCS benefit, which is now leading to international acceptance of lung cancer screening, Dr. von Eschenbach was presented the 2019 James L. Mulshine, MD, Leadership Award (Fig. 1). Dr. von Eschenbach's remarkable career involved working over 3 decades as a physician, surgeon, oncologist, and executive, including serving as Chairman of the Department of Urologic Oncology, Executive Vice President, and

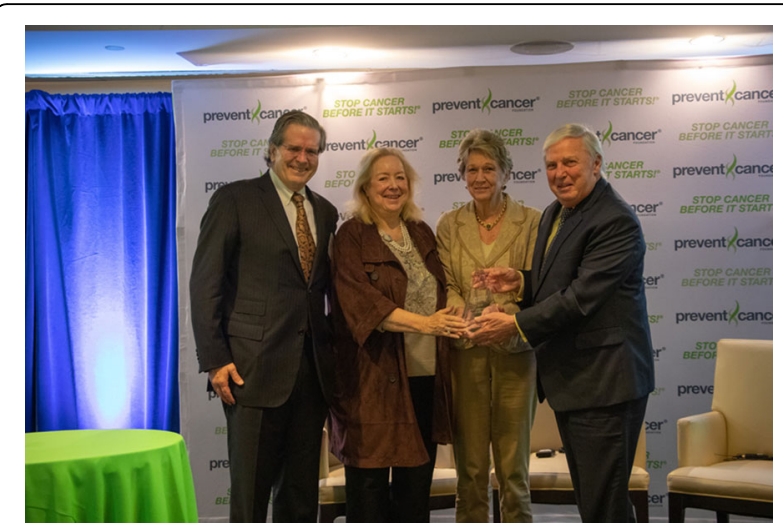

Fig. 1 Recently honored Andrew C. von Eschenbach, M.D., with the 2019 James L. Mulshine, M.D., Leadership Award for his work leading the National Lung Screening Trial (NLST), the first largescale clinical trial for low-dose computed tomography $(\mathrm{CT})$ lung cancer screening. The award was presented on October 10, 2019, at the 16th annual Quantitative Imaging Workshop: Lung Cancer, COPD, and Cardiovascular Disease. Andrew C. von Eschenbach, M.D., receives the James L. Mulshine, M.D., Leadership Award from the Prevent Cancer Foundation. Left to right: Dr. Mulshine; Claudia Henschke, Ph.D., M.D.; Carolyn R. Aldigé; and Dr. von Eschenbach

Chief Academic Officer at the University of Texas MD Anderson Cancer Center in Houston. He also served as President-elect of the American Cancer Society. After his time as NCI Director, he went on to serve as the Commissioner of the U.S. Food and Drug Administration (FDA).

\section{Current status of lung cancer screening management}

During the workshop, critical discussions examined the evolution of risk biomarkers for early lung cancer management. To date, despite many trials using molecular epidemiology approaches, none of the candidate molecular markers has shown clinical utility beyond the established metrics of age and tobacco exposure in predicting lung cancer risk in tobacco-exposed populations [14].

Interest has also grown in the use of modeling approaches to evaluate risk in heavily tobacco-exposed populations. The evolution of a modeling effort was discussed to define the mortality benefit resulting from using different CT imaging time intervals for ongoing lung cancer screening using models [15]. Investigators from Rice University have built models based on existing screening trial results found to improve outcomes with different intervals of serial repeat screening analysis. In this effort and only using recent CT screening experience outcomes to build and validate the model (rather than the older validation data derived from chest X-ray screening data). Dr. Olga Gorlova's preliminary results suggest that annually- repeated LCS gives the most favorable mortality reduction benefit and coincides with the recently published screening interval experience 
from the NELSON trial $[10,16]$. Further, the Gorlova model demonstrates significant additional mortality reduction benefit resulting from continuing annual CT screening beyond the two annual screening cycles which were the basis for the NLST results. This prediction is now supported by long term follow-up of a number of major international screening efforts which have also reported favorable mortality reductions, considerably in excess of the NLST findings [10-13]. While such information is slow to percolate into the shared decisionmaking discussions around benefits/harms of participating in lung cancer screening, this lag may in part account be responsible for the tepid public acceptance of LCS. Yet, the collective impact of the favorable outcomes reported with the more recent screening reports may constitute an inflection point in the acceptance of lung cancer screening as a robust tool for improving tobacco-related adverse consequences. If this is true and annual repeat LDCT is performed on growing numbers of heavily tobacco-exposed individuals, it has important implications for the CT imaging service that will be the basis of this screening management. For example, quality measures to ensure the reliability of measured annual changes in nodule volume will be required to ensure responsible clinical management of the screening subjects.

\section{Evolving trends in integrating quantitative CT imaging into LCS}

This workshop series started in 2004 to facilitate the introduction of quantitative CT imaging to improve early lung cancer detection and treatment [17]. In the years since the first workshop, there has been considerable progress in optimizing visualization of early lung cancer with low dose CT. In 2007, the Radiological Society of North America developed a broad consortium of interested parties called the Quantitative Imaging Biomarker Alliance (QIBA) to optimize quality provisions for all types of quantitative medical imaging. This effort included a focus on early lung cancers imaging within the context of LCS [18-20]. Over time, there have been extensive interactions between QIBA and the PCF Workshop leadership to address the shared goal of advancing quantitative CT imaging as a tool for early lung cancer management.

Precise quantitative imaging for pulmonary nodules with volumes between 5 and $12 \mathrm{~mm}$ in diameter required for successfully screening for early, curable lung cancer is a demanding challenging task [18]. The advantages of CT in this setting includes high spatial resolution even using low medical radiation dose for image acquisition. In regard to the cost of a thoracic CT for lung cancer screening, current federal reimbursement for performing and reading a CT for lung cancer screenings is less than $\$ 250$. In regard to feasibility, this study can be acquired painlessly requiring much less than a minute to acquire a comprehensive study. Finally, there are currently already deployed a surplus of CT scanners available across many communities in the United States that are currently being utilize to enable implementation of this new cancer screening service.

From the field work done in developing the conformance process for optimal characterization of small non calcified pulmonary nodules, resolution through the entire field of view emerged as the main imaging parameter determining nodule measurement precision [18]. Of note, ensuring optimal image resolution throughout the full field of view is critical to allow for accurate characterization of pulmonary nodules in the peripheral regions of the lung as that is where lung cancers frequently arise.

The technical and metrological issues embedded in reliably imaging small image volumes for detecting early stage lung cancers in the range of nodule volumes from 5 to $12 \mathrm{~mm}$ in diameter are formidable. In screening management, when a $6 \mathrm{~mm}$-in-diameter nodule grows to $7 \mathrm{~mm}$ in diameter, visually it may seem like a small change. In reality, this represents a $50 \%$ increase in the volume of the nodule. For LCS, the clinical objective is frequently to detect a volume change in suspicious lung nodules over a time-interval. This task routinely occurs with annual LCS visits. Precise measurement of lung nodules can be thwarted by the inherent complexities of the thoracic anatomy related to the confluence of bony, muscular, and vascular structures all moving within the confines of the chest. In addition, each vendor employs its own proprietary image processing approach making cross imaging platform comparisons complex. Then, there is an array of technical factors, such as variations in subject positioning on the imaging table, that can change each time a subject is imaged.

A recent example of this collaboration has been a QIBA research project supported by the PCF to provide low-cost imaging phantoms (CTLX-1) that were developed specifically to evaluate the quantitative functioning CT scanners to measure small thoracic nodules for LCS [21]. QIBA had defined parameters for optimal measurement of suspicious lung nodules for early lung cancer detection at LCS [18]. The volume range of these lesions of interest for LCS ranges from $5 \mathrm{~mm}$ in diameter to just over a centimeter. Consistent and precise measurements in a volume range that small are beyond the bounds of usual clinical care imaging. Therefore, to address the image acquisition quality, LCS sites can upload a CT-acquired image of the CTLX-1 phantom using their proposed LCS imageacquisition protocol via a dedicated, QIBA-hosted, cloud-based infrastructure. The transmitted phantom image is rapidly analyzed per the QIBA conformance process to characterize the actual performance of the 
site's CT scanner based on the fidelity of its transmitted image to the actual known physical characteristics of the CTLX phantom [21]. This process has been undertaken by 100 LCS sites from across the world, testing hundreds of individual scanners. Comprehensive analysis of these images reveals that most LCS sites employ CT image acquisition parameters that do not allow optimal nodule measurements due to compromise in image resolution or other critical characteristics. However, the automated phantom analysis can often identify simple alterations in the CT image acquisition parameters that can be transmitted back to the LCS site, so that revised acquired CT image achieves the level of measurement performance to enable accurate volume measurement. Based on these efforts, this image quality resource is now globally available from QIBA as a hosted, web-based service to enable improvement to low-dose CT image acquisition across the world [18].

In moving to clinical application, defining reliable levels of imaging measurement performance requires establishing processes to independently validate the actual quality of quantitative imaging biomarkers. Then such approaches must be disseminated to allow responsible integration of these validation tools to support relevant clinical decision making. The FDA has recently proposed an approach to optimizing quantitative imaging measurement quality for medical devices https://www.fda. gov/media/123271/download, accessed July 15, 2020. The FDA's intention is to facilitate the development of standards and validation tools to ensure the precise performance of tools used in medical-related processes. Their approach to process standards emphasizes achieving meaningful performance levels and allowing innovators to define how such performance is achieved. Having explainable elements may prove beneficial over time in terms of allowing inter-operability of imaging platforms, as there has been a productive experience with consensus approaches such as with efforts to define image measurement metrology and digital imaging and communications in medicine (DICOM) data format $[22,23]$.

\section{Extracting all available information from thoracic imaging of tobacco-exposed individuals}

A shared goal of many researchers is to evaluate high resolution, 3-D thoracic CT lung images under a single framework to assess the three leading causes of premature death: lung cancer, chronic obstructive pulmonary disease (COPD) and coronary artery disease (CAD) due to shared pathogenesis related to tobacco exposure [24]. These comorbid conditions are frequently evident in the thoracic CT scan of individuals with heavy tobacco exposure as the peak incidence of these three diseases are in the same age range as is the current eligibility for lung cancer screening.

It is now well reported that there are frequent findings of COPD while performing lung cancer screening [25]. Many individuals being screened for lung cancer under the current USPSTF guidelines will be found to have COPD and initial efforts to ensure the quality of LDCT imaging must be also evaluated to ensure those conditions do not compromise efforts to characterize COPD status. Separate efforts are ongoing within the QIBA to define the most productive diagnostic approach for CT imaging of chronic obstructive pulmonary disease, and an independent profile document for this type of CT imaging has already been developed (https://qibawiki. rsna.org/index.php/Lung_Density_Biomarker_Ctte). The work to develop this profile has helped to define the critical elements of COPD imaging that must be preserved in the setting of LCS so that both diseases can be optimally detected. Management of COPD in the context of LCS was presented by Dr. Lee Gazourian. The Lahey Hospital and Medical Center has spearheaded the use of quantitative imaging to diagnose COPD in their LCS population. They showed an association with the risk of COPD hospitalizations and how this information can be utilized to manage this at-risk population, reduce cost and improve outcomes using a reported bestpractice clinical path program [26].

Recently, coronary calcium scoring has emerged as the most informative predictor of cardiac risk [27]. It is now well established that coronary calcium can be routinely visualized when obtained through a thoracic CT scan for LCS [28]. Morteza Naghavi, MD, reviewed the public health implications of the detection of coronary calcium, including by thoracic CT imaging. Dr. Naghavi is a cardiologist who advocates for the broader use of coronary calcium imaging as a preventive biomarker for managing cardiac mortality risk. To advance this work, Dr. Naghavi established the Society of Heart Attack Prevention and Eradication as a forum to advance the use of coronary calcium analysis including in the setting of LCS [29].

Performing thoracic $\mathrm{CT}$ imaging for lung cancer and COPD as well as coronary calcium has become a major focus of this Workshop, to allow the full public health benefit of thoracic CT imaging as a "smoking-related diseases" screening tool [24]. Epidemiologists have reported that although lung cancer cases will substantially decline between now and 2065, over 4.4 million people will still die of lung cancer and about 20 million adults of ages 30-84 will continue to smoke in 2065 [30]. Given the vast numbers of individuals sharing health risks related to tobacco exposure, current budget pressures, considerations of efficiency and economy are critical, especially in addressing 
equity concerns in assuring screening access along with tobacco cessation services as needed by members of medically underserved communities.

Currently, these image quality tools are also being implemented in an ongoing effort of the United States Department of Veterans Affairs (VA) in which they are working to develop an integrated, open-source software clinical management tool for routine clinical care at VA facilities, given their large population of tobacco-exposed individuals. Dr. Claudia Henschke of Icahn School of Medicine at Mount Sinai and Rick Avila are co-principal investigators of this effort along with Dr. Drew Moghanaki of the Atlanta VA. This software environment leverages the vast experience the International Early Lung Cancer Action Project (I-ELCAP) accrued in efficiently managing the screening experience of over 70,000 screening subjects over the last two decades [31]. As demonstrated in a report of diagnostic work-up rates among I-ELCAP participants, the frequency of full diagnostic work-ups could be greatly reduced by restricting diagnostic work-up only to individuals with pulmonary nodules with a diameters $6 \mathrm{~mm}$ and greater [32]. In this way, the risks associated with misdiagnosis can be overcome by using a defined quantitative measurement method that result in more consistently accurate nodule measurement $[31,32]$.

To realize this vision, further efforts are needed to ensure that best quality approach to thoracic imaging is used to assess for these three major thoracic diseases, performed in a fashion that ensures reliable quantitative assessment for each of the three disease sites. Currently, there is no consensus approach to this, to establish how such an integrating imaging approach should be performed as the assessment of these three diseases has been quite different. However, this is a favorable time to attempt such an ambitious approach as CT detector technology, image analysis approaches and radiomics have all undergone rapid improvement. In addition, artificial intelligence (AI) approaches are just being applied in the thoracic imaging space and thoracic screening is an opportunity where AI may bring considerable benefit.

\section{Artificial intelligence in LCS}

A critical focus for the more quantitative-oriented participants at the workshop explores the issue of aligning quantitative thoracic CT imaging with artificial intelligence (AI) research to identify strategies for accelerating progress in this exciting new field [33]. Discussions explored the possibility of educating clinicians on how they may use AI to enhance their productivity or to improve clinical outcomes. The group also considered what new competencies are required to prepare lung screening professionals to allow faster and better uptake of such new tools. There are also additional areas with imaging process optimization could have benefit.

Over the course of the Quantitative Imaging Workshop series, there have been ongoing efforts to create large, high quality, and open CT image databases to enable not only imaging tool development but also robust validation. However, despite considerable effort, the rapid pace of improvement with imaging technology has limited the utility of existing thoracic CT databases for cutting edge research efforts [34-36]. Virtually all existing public lung cancer imaging databases were developed before the maturation of CT lung cancer screening that utilizes the current, higher resolution imaging technology. Now that the reality of AI is emerging in many fields, this gap has become more critical. To support rapid and responsible image database development, some workshop participants recommended establishing a standardized approach for a patient to declare that their data may be used for scientific research including AI development. Such a global opt-in approach could help remove many of the barriers that exist in making large open CT lung imaging databases a reality.

Establishing a large and well curated image archive of recent CT scans could also help improve the evidence foundation for CT image quality efforts such as the QIBA CT small lung nodule profile [18]. Specifically, the archive could facilitate better approaches to measuring part-solid lung nodules. These putative lung cancer precursor lesions currently have higher measurement variability due to lower contrast between the lung parenchyma and the solid component of a part-solid nodule and so this constitutes a more complex measurement challenge.

In addition, a large image archive could facilitate developing more precise methods of ensuring inter-operability of serial module measurements. At the present time, there is considerable variance with volumetric measurement of thoracic CT images obtained on two different types of CT scanners using comparable imaging protocols in the course of routine, annual LDLC screening. Success with precisely measuring lung nodules across different combinations of CT imaging platforms could simplify existing logistical burden in arranging annual follow-up scans for screening subjects being scanned on different types of CT scanners.

Considerations with imaging informatics tool development were brought into sharp focus with the presentation of Philip Hoelzer from Siemens Healthineers. In discussing the capabilities of the new Siemens lung cancer screening software tool, which included the first application of artificial intelligence, Dr. Hoelzer emphasized the importance of working with clinical radiologists in real-world settings to ensure seamless integration of new imaging informatics tools within routine clinical workflows to support broad adoption 
of these new tools without disrupting the efficient delivery of clinical care.

\section{Moving forward}

Realizing the full potential of thoracic CT screening, while promising, still involves addressing challenging issue. The implementation of lung cancer screening alone has faced headwinds despite considerable new and strongly validating evidence of robust benefit and modest harms [37]. Could implementation of lung cancer screening implementation be hindered due to concerns with the complex management of two other leading causes of death (CAD and COPD) in this heavily tobacco-exposed cohort? Fortunately, this appears unlikely as integrating care for the three leading causes of tobacco-related chronic disease is already happening at leading screening centers [38]. A more inclusive approach with thoracic CT imaging in tobacco-exposed cohorts will be disseminated through existing support for lung cancer screening, which is already federally reimbursed. In this fashion, routinely acquiring additional information about the two other leading causes of premature death (COPD and heart disease) can be done at an incremental cost within the screening effort. Leading screening programs already have considerable experience in performing this multi-disease screening, with clinicians already integrating this complex imaging information into productive discussions with individuals undergoing such screening [38].

To responsibly sustain such an evolution, it becomes even more important to follow screened populations for clinical outcomes. Collections of thoracic CT images obtained can also enable screening quality control evaluations that can be used to accelerate image tool development for the spectrum of tobacco-related thoracic diseases. Develop robust quantitative imaging software tools for efficient and economical clinical management for pre-symptomatic, high risk populations is an unrecognized unmet need. Pragmatically, the greatest barrier to producing such imaging tools with existing approaches, is the cost that companies would have to pay to collect sufficient relevant imaging information. A major discussion at the workshop was the possibility of engaging the public to support the re-use of acquired thoracic CT images from routine screening care to fast- track the development and validation of integrated screening imaging tools for lung cancer, coronary arteries and lung parenchyma.

From the in-depth discussions around this issue, it will be critical to work with advocates and professional societies to communicate to the public the importance of the thoracic screening opportunity and the catalytic value of image and data donations in realizing this public health opportunity. There was strong consensus that to accomplish these important goals, a major communications campaign is essential to raise public awareness around the overall benefits of screening as a health check and not simply screening for lung cancer.

In addition, currently the challenge with LCS uptake is not only with numbers but also with the issue of equity. As the use of this service rises, will all elements of society benefit from these powerful new imaging tools equally [39]? Remarkable progress has also been reported with improving colon cancer uptake rates especially in extending benefit of this approved cancer screening service to medically underserved populations. A best practice in this regard is the implementation of cancer screening in the state of Delaware [40, 41]. Dr. Al Rizzo outlined how in Delaware after a protracted inter professional effort, they adopted a state-wide program of nurse navigators for other, non-lung, cancer screening outreach including in medically underserved communities $[40,41]$. Their system empowered functional committees with state-wide representation from all health systems to implement a bottom-up approach to map out effective screening delivery coupled with comprehensive metrics to guide the evolution of the screening effort. This effort resulted in a high screening participation across the defined target population, including medically underserved communities. These approaches are now being applied to LCS services in that state.

Learning from previous screening efforts, there is a fundamental need to effectively communicate the value of the screening service, but also to establish a scalable delivery model across diverse screening communities for the efficient delivery of this public health service [42]. Therefore, in addition to refining imaging tools to optimally characterize all three thoracic tobacco-related diseases, there is a critical need to invest in clinical decision-support and dissemination tools to ensure efficient clinical delivery of the integrated screening process in real-world settings.

Readily available, workflow-friendly screening management tools are not yet at hand to ensure busy clinicians are efficiently recording critical information which would be the basis for tailored communications tools. These tools will be essential to allow already bandwidthrestricted clinicians to effectively and reliably communicate with screening subjects and sustain this thoracic screening process. This is a significant and largely ambulatory care coordination challenge. Tasks include assisting clinicians both in the in-take referral of patients to the screening program as well as with communication around scheduling, providing results and then determining action plans. Reflecting the current screening literature, there were extensive discussions about the role of such navigator/facilitators with this new model of care which is emerging to implement high quality lung cancer screening. A practical implication is that the imaging 
management process has to be integrated into the comprehensive screening subject management software to ensure that optimal outcomes occur.

Such integration of quantitative imaging results that is now emerging as a key part of the LCS management process will also be equally relevant to support the integrated care for the other two major thoracic CTdetected diseases (COPD and CAD). To accomplish this integrative care will entail coordination of technical imaging factors such as defining a single image acquisition protocol to optimally evaluate across three distinct disease entities. Further, it is possible that we will learn that the best image acquisition approach may differ relative to the needs of specific populations as potentially related to shared xenobiotic exposures and genetic background. Attention to such issues going forward will be critical, but the consensus was that to begin this approach, a defined high-quality overall protocol should be implemented. Then, through time, analysis of outcomes may improve performance by refining the image acquisition parameters. Currently, the QIBA Small Lung Nodule profile proposes a best practice, standardized image acquisition protocol for lung cancer screening which will be incorporated as the default image acquisition approach. More research is required to best define default standard data reporting elements for quantitative imaging endpoints as well as standardized management protocols for specific clinical findings.

A potential benefit of improving LDCT screening in pre-symptomatic cohorts of tobacco-exposed individuals is that many more people will be identified in the near term with early, asymptomatic lung cancer, COPD and CAD with existing state-of-the-art imaging and computational tools [43]. A sustained focus of the workshop has been to encourage the pharmaceutical industry to develop new therapeutics for these early, screen-detected illnesses. Likewise, the LCS setting is also equally committed to encouraging the use of preventive measures including improvements in diet, exercise and smoking cessation as relevant. Maintaining continuous innovation in this sector to allow progress in the development of effective preventive agents as well as development of public health measures to ensure equitable access to these services merits ongoing financial investment.

Nationally, it is a time of large-scale transition to a population health. Therefore, to support LCS implementation, it is also critical to address the issue of ensuring reimbursement for these new and evolving screening management services. However, to sustain reimbursement there also has to be an objective measure of health benefit. Therefore, discussions included consideration of what will be the cohesive national quality process to ensure the value of integrating quantitative imaging processes into LCS.
The benefits of holistic integration and the metrology of image quality improvement may represent two ends of a spectrum of healthcare management. In the middle of that spectrum are the payer population health metrics, which shift billions of dollars of healthcare funds and have been instrumental in improving cancer screening rates, diabetes care, and immunization rates, among other outcomes. However, there are no payer metrics for LCS. Today, payers have no incentives to encourage LCS.

For example, Medicare uses Star metrics based on HEDIS (Healthcare Effectiveness Data and Information Set) measures to adjust payments to Medicare Advantage plans, which now enroll close to $40 \%$ of Medicare beneficiaries ( 25 million lives), as well as payments to Part D plans (close to 50 million lives) and Accountable Care Organizations (12 million lives). Similar metrics are used to shift funds among Affordable Care Act Marketplace plans, and to reward / penalize Medicaid Managed Care Organizations. Commercial insurers are also measured on similar metrics. Consequently, the creation and adoption of one or more HEDIS metric would be an incredibly powerful advance for LCS. Basically, payers would receive financial incentives/penalties to promote LCS for their members. For existing HEDIS measures, payers implement programs to meet their targets, and they have an interest in implementing programs that will produce the desired scores.

The HEDIS measures for cancer screening would seem to be a useful starting point. These measures are ratios-the number of eligible members who were screened in a defined period divided by the total number of members who were eligible for screening. However, there is a significant challenge for a LCS HEDIS measure, which is that there are no reliable ways to establish a payer's denominator-the number of members eligible for screening. This is because the data on an individual's smoking history is not reliably collected for a payer's membership, and when it is collected, it is not readily available. Furthermore, personal attestations of smoking history have proved to vary from one interview to another.

Ideally, HEDIS measures can be produced entirely with administrative data--medical claims. This is because there is no other way to quickly, repeatedly, and inexpensively generate current information for tens of millions of lives. HEDIS scores are produced annually.

Consequently, in Workshop discussions over the last 2 years, we have proposed a HEDIS metric based on a yield rate-the portion of incident lung cancer cases in a year that were found through screening. That is, the numerator is the number of new, screen-detected lung cancers, and the denominator is the number of incident lung cancers. There are a number of details to work out, and the different possible algorithms that can produce 
this ratio would need to be tested for reasonability, reliability and stability.

The National Committee on Quality Assurance administers HEDIS and has a standard process for creating and evaluating new measures. The development of a HEDIS measure for LCS should be a priority for LCS advocates.

In closing, a range of opportunities to improve the imaging processes with LCS are outlined in Table 1. Innovations with both $\mathrm{CT}$ scanning capabilities and image processing have been occurring rapidly for a number of years. To understand the quality of imaging in "real world" LCS settings, it is critical to prospectively acquire images obtained in the screening process, so that the performance of image measurements can be determined objectively. With new thoracic CT image collections as well as with established image collections, there is a need to support curation of the image collections to ensure that they are a reliable source of "ground truth". To establish truth may involve expert radiology review or systematic review with define software tools. In this fashion, validation with imaging biomarkers can proceed

Table 1 Consensus aspirations for advancing quantitative imaging to improve lung cancer screening and quantitative tool development and validation

1) Create a public database of 50,000 lung cancer screening imaging datasets

- Leverage available data and phantom validation tools to ensure this is a high-quality image collection.

•Ensure that there is sufficient diversity (e.g., demographic, geographic, disease states, scanner models).

-Ensure that major lung cancer sub-populations (e.g., small cell) are represented in such a collection.

-Provide reliable sources of "Ground truth" information including lung nodule biopsy results.

2) Increase resource to support and curate new and existing databases and related technology infrastructure

-Centralized Database Example: The Cancer Imaging Archive (TCIA)

-Distributed Database Example: Early Lung Imaging Confederation (ELIC).

-Technology Example: Automated insertion of lung nodules in existing CT scans.

3) Investigate a New Global Patient Data Electronic Submission Infrastructure

-Allow global patients to opt-in to having their data used by researchers with appropriate de-identification.

-Hospitals and healthcare facilities will inform patients of this opportunity to advance research.

-A global opt-in strategy will need informed consent templates for different regions of the world.

4) Improve the QIBA Small Lung Nodule Profile

-Add support for volume measurement of part-solid lung nodules.

-Add support for the ability to use multiple $C T$ scanners over time to measure lung nodules. in a much more rigorous and standardized fashion. To ensure ongoing collections of thoracic images, the public has to be informed on how these resources are required for the development of more capable image measurement tools that may provide the foundation for further innovation with complex detection and management tools such as with AI. Administrative mechanisms are required to ensure the integrity of the data security measures as well as to make the donation of medical images for research more commonplace. This may entail innovative software development to reduce the workflow burden of obtaining such image donations even in very busy, ambulatory imaging centers with limited capacity to support research efforts.

Finally, these new and precise imaging tools applied in clinical settings such as with small pulmonary nodule measurement for LCS may be foundational tools in population health settings with other thoracic, tobaccorelated diseases, where their application can have major strategic importance in enabling significantly impact such as in reducing mortality burden.

\section{Conclusion}

LCS is an effective cancer screening process that benefits from rapid advances in the quantitative assessment of early lung cancer as well as computer-driven analysis capabilities. As such LCS is a prime target for realizing the promise of AI. However, to ensure responsible evolution, as discussed, there are complex layers of development that can benefit from coordination. As tobaccorelated thoracic diseases are such prominent public health issues, important policy issues are also relevant, including health equity and reimbursement. This meeting report summarizes the cross-cutting conversations of a diverse array of professionals as they consider how to continue to make progress in the application of advanced imaging to intercept the progression of an important set of major chronic intrathoracic diseases.

\section{Abbreviations}

PCF: Prevent Cancer Foundation; LCS: Lung cancer screening: NCl: National Cancer Institute; NLST: National Lung Screening Trial; FDA: Food and Drug Administration; NELSON: Nederlands-Leuvens Longkanker Screening Onderzoek; QIBA: Quantitative Imaging Biomarker Alliance; DICOM: Digital Imaging and Communications in Medicine; COPD: Chronic obstructive pulmonary disease; CAD: Coronary artery disease; VA: United States Department of Veterans Affairs; I-ELCAP: International Early Lung Cancer Action Project; Al: Artificial Intelligence; TCIA: The Cancer Imaging Archive; ELIC: Early Lung Imaging Confederation; HEDIS: Healthcare Effectiveness Data and Information Set

\section{Acknowledgements}

We appreciate the diligent technical support of Liz Hall.

\section{Authors' contributions}

All authors were involved in the conceptualization of the meeting and the resulting report. All authors were involved with the writing and editing of this document. Author's Permission to Submit: All of the above authors have agreed to submit this manuscript for publication. 


\section{Funding}

Not applicable.

\section{Availability of data and materials Not applicable.}

\section{Ethics approval and consent to participate}

Not applicable.

\section{Consent for publication}

Not applicable.

\section{Competing interests}

James L. Mulshine, M.D. - No conflicts with the contents of this submission. Rick Avila, MS - Mr. Rick Avila is a co-owner and CEO of Accumetra, LLC, a private company which develops and provides technology to improve the quality of CT imaging including calibration phantoms mentioned within this paper

Daniel C. Sullivan, M.D. - No conflicts with the contents of this submission. David F. Yankelevitz, M.D. - Dr. David Yankelevitz is a named inventor on a number of patents and patent applications related to the evaluation of diseases of the chest including measurement of nodules. Dr. Yankelevitz has received financial compensation for licensing of these patents. In addition, he is a consultant for and co-owner of Accumetra, a private company developing tools to improve the quality of CT imaging and is on the medical advisory board for Grail, a company that does blood tests for early detection of cancer.

Raul San Jose Estepar, Ph.D. - Dr. Raul San Jose Estepar is co-founder and advisor of Quantitative Imaging Solutions, a private consulting company for imaging analytics and visualization.

Laurie Fenton Ambrose - No conflicts with the contents of this submission. Bruce Pyenson, FSA, MAAA - No conflicts with the contents of this submission.

Carolyn R. Aldigé - No conflicts with the contents of this submission.

\section{Author details}

${ }^{1}$ Rush University, Chicago, IL, USA. ${ }^{2}$ Accumetra, LLC, Clifton Park, NY, USA. ${ }^{3}$ Duke University, Durham, NC, USA. ${ }^{4}$ Icahn School of Medicine, The Mount Sinai Health System, New York, NY, USA. ${ }^{5}$ Brigham and Women's Hospital Harvard Medical School, Boston, MA, USA. ${ }^{6} \mathrm{GO} 2$ Foundation for Lung Cancer, Washington, DC, USA. ${ }^{7}$ Milliman, Inc., New York, NY, USA. ${ }^{8}$ Prevent Cancer Foundation, Alexandria, VA, USA.

Received: 27 August 2020 Accepted: 21 September 2020 Published online: 27 October 2020

\section{References}

1. Henschke Cl, McCauley DI, Yankelevitz DF, et al. Early Lung Cancer Action Project: overall design and findings from baseline screening. Lancet. 1999; 354(9173):99-105. https://doi.org/10.1016/S0140-6736(99)06093-6 PMID: 10408484

2. Yankelevitz DF, Gupta R, Zhao B, Henschke Cl. Small pulmonary nodules: evaluation with repeat CT--preliminary experience. Radiology. 1999;212:561-6.

3. van Klaveren RJ, Oudkerk M, Prokop $M$, et al. Management of lung nodules detected by volume CT scanning. N Engl J Med. 2009:361(23):2221-9. https://doi.org/10.1056/NEJMoa0906085 PMID: 19955524

4. Field JK, Duffy SW, Baldwin DR, et al. The UK lung cancer screening trial: a pilot randomized controlled trial of low- dose computed tomography screening for the early detection of lung cancer. Health Technol Assess. 2016;20(40):1-146. https://doi.org/10.3310/hta20400 PMID: 27224642

5. Aberle DR, Berg CD, Black WC, et al. The national lung screening trial: overview and study design. Natl Lung Screen Trial Res Team Radiol. 2011; 258(1):243-53. https://doi.org/10.1148/radiol.10091808 Epub 2010 Nov 2. PMID: 21045183

6. National Lung Screening Trial Research Team, Aberle DR, Adams AM, Berg $C D$, et al. Reduced lung-cancer mortality with low-dose computed tomographic screening. N Engl J Med. 2011;365(5):395-409. https://doi.org/ 10.1056/NEJMoa1102873 Epub 2011 Jun 29. PMID: 21714641.

7. Printz C. Lung cancer alliance praises CMS decision to cover lung cancer screenings. Cancer. 2015;121(8):1157. https://doi.org/10.1002/cncr.29376 PMID: 25855332
8. Centers for Medicare \& Medicaid Services. Decision memo for screening for lung cancer with low-dose computed tomography (LDCT). https://www. cms.gov/newsroom/press-releases/national-coverage-determination-ncdscreening-lung-cancer-low-dose-computed-tomography-ldct. [Accessibility verified 2 June 2020].

9. National Lung Screening Trial Research Team. Lung cancer incidence and mortality with extended follow-up in the National Lung Screening Trial. J Thorac Oncol. 2019;14(10):1732-42. https://doi.org/10.1016/j.jtho.2019.05.044 Epub 2019 Jun 28. PMID: 3126083.

10. de Koning $\mathrm{HJ}$, van der Aalst CM, de Jong PA, et al. Reduced lung-cancer mortality with volume CT screening in a randomized trial. N Engl J Med. 2020;382(6):503-13. https://doi.org/10.1056/NEJMoa1911793 Epub 2020 Jan 29. PMID: 31995683.

11. Pastorino $U$, Silva $M$, Sestini $S$, et al. Prolonged lung cancer screening reduced 10-year mortality in the MILD trial. Ann Oncol. 2019. https://doi. org/10.1093/annonc/mdz117 [Epub ahead of print] PMID: 30937431

12. Nawa T, Fukui $K$, Nakayama $T$, et al. A population-based cohort study to evaluate the effectiveness of lung cancer screening using low-dose $\mathrm{CT}$ in Hitachi city, Japan. Jpn J Clin Oncol. 2019;49(2):130-6. https://doi.org/10. 1093/jjco/hyy185.

13. Markowitz SB, Manowitz A, Miller JA, et al. Yield of Low-Dose Computerized Tomography Screening for Lung Cancer in High-Risk Workers: The Case of 7189 US Nuclear Weapons Workers. Am J Public Health. 2018;108(10):1296302. https://doi.org/10.2105/AJPH.2018.304518 Published online 2018 October. PMCID: PMC6137787. PMID: 30138066.

14. Seijo LM, Peled N, Ajona D, et al. Biomarkers in lung cancer screening: achievements, promises, and challenges. J Thorac Oncol. 2019;14(3):343-57. https://doi.org/10.1016/j.jtho.2018.11.023 Epub 2018 Dec 4. PMID: 30529598.

15. Chen X, Foy M, Kimmel M, Gorlova OY. Modeling the natural history and detection of lung cancer based on smoking behavior. PLoS One. 2014;9(4): e93430. https://doi.org/10.1371/journal.pone.0093430 eCollection 2014. PMID: 24705368

16. Yousaf-Khan $U$, van der Aalst $C$, de Jong PA, et al. Final screening round of the NELSON lung cancer screening trial: the effect of a 2.5-year screening interval. Thorax. 2017;72(1):48-56. https://doi.org/10.1136/thoraxjnl-2016208655 Epub 2016 Jun 30. PMID: 2736464044.

17. Mulshine $\mathrm{J}$, Avila RS, Hirsch FR, Yankelevitz D. Developing CT imageprocessing tools to accelerate progress in lung cancer drug development. Oncology (Williston Park). 2006;20(12):1606-1608-10.

18. Radiological Society of North America- Quantitative Imaging Biomarker Alliance Wiki. https://qibawiki.rsna.org/images/b/b3/QIBA_CT_Vol_ SmallLungNoduleAssessmentInC5354TScreening_2018.11.18-clean-3.pdf. Accessed 2 Aug 2020.

19. Mulshine JL, Gierada DS, Armato SG 3rd, et al. Role of the quantitative imaging biomarker alliance in optimizing $C T$ for the evaluation of lung cancer screen-detected nodules. J Am Coll Radiol. 2015;12:390-5.

20. Rydzak CE, Armato SG, Avila RS, et al. Quality assurance and quantitative imaging biomarkers in low dose CT lung cancer screening. $\mathrm{Br} J$ Radiol. 2017; [Epub ahead of 1516 print]. [Crossref] [PubMed]. McNeil C. Low-Dose CT Lung Screening: New Developments Support Increased Quality.

21. More Data, Deep Learning ASCO Post. https://www.ascopost.com/issues/ december-25,2018/low-dose-ct-lung-screening/, Accessed on 8 Jul 2020.

22. Sullivan DC, Obuchowski NA, Kessler LG, et al. RSNA-QIBA metrology working group. Metrology standards for quantitative imaging biomarkers. Radiology. 2015;277(3):813-25. https://doi.org/10.1148/radiol.2015142202 Epub 2015 Aug 12. PMID: 2626783.

23. Maglogiannis I, Andrikos C, Rassias G, Tsanakas P. A DICOM based collaborative platform for real-time medical teleconsultation on medical images. Adv Exp Med Biol. 2017;989:79-91. https://doi.org/10.1007/978-3319-57348-9_7 PMID: 28971418.

24. Mulshine JL. One screening for ischemic heart disease, lung cancer, and chronic obstructive pulmonary disease: a systems biology bridge for tobacco and radiation exposure. Am J Public Health. 2018;108(10):1294-5. https://doi.org/10.2105/AJPH.2018.304655 Published online 2018 October. PMCID: PMC6137757. PMID: 30207781.

25. Seijo LM, Zulueta JJ. Understanding the Links between lung cancer, COPD, and emphysema: a key to more effective treatment and screening. Oncology (Williston Park). 2017;31(2):93-102 PMID: 28205188.

26. Bourbeau J, Julien M, Maltais F, et al. For the chronic obstructive pulmonary disease axis of the respiratory network Fonds de la Recherche en Santé du 
Québec reduction of hospital utilization in patients with chronic obstructive pulmonary disease: a disease-specific self-management intervention. Arch Intern Med. 2003;163(5):585-91. https://doi.org/10.1001/archinte.163.5.585.

27. McClelland RL, Jorgensen NW, Budoff $M$, et al. 10-year coronary heart disease risk prediction using coronary artery calcium and traditional risk factors: derivation in the MESA (Multi-Ethnic Study of Atherosclerosis) with validation in the HNR (Heinz Nixdorf Recall) Study and the DHS (Dallas Heart Study). J Am Coll Cardiol. 2015;66(15):1643-53. https://doi.org/10. 1016/j.jacc.2015.08.035 PMID: 26449133. PMID: 20829542.

28. Shemesh J, Henschke Cl, Shaham D, et al. Ordinal scoring of coronary artery calcifications on low-dose CT scans of the chest is predictive of death from cardiovascular disease. Radiology. 2010;257(2):541-8. https://doi.org/10.1148/ radiol.10100383 Epub 2010 Sep 9.

29. Naghavi M, Maron DJ, Kloner RA, et al. Coronary artery calcium testing: a call for universal coverage. Prev Med Rep. 2019;15:100879. https://doi.org/ 10.1016/j.pmedr.2019.100879 eCollection 2019 Sep. PMID: 31193256.

30. Jeon J, Holford TR, Levy DT, et al. Smoking and lung cancer mortality in the US from 2015-2065: a comparative modeling approach. Ann Intern Med. 2018. https://doi.org/10.7326/M18-1250 Published online 2018 Oct 9. PMC D: PMC6242740 NIHMSID: NIHMS992587.PMID: 30304504.

31. Henschke Cl, Yankelevitz DF, Reeves AP, Yip R. Evolution of lung cancer screening management. Oncology (Williston Park). 2019;33(7):629380 PMID: 31365749 .

32. Henschke Cl, Yip R, Yankelevitz DF, Smith JP, for the I-ELCAP Investigators. Definition of a positive test result in computed tomography screening for lung cancer: a cohort study. Ann Internal Med. 2013;158:246-52 PMID: 23420233.

33. Matheny ME, Whicher D, Thadaney IS. Artificial Intelligence in Health Care: a report from the National Academy of Medicine. JAMA. 2019. https://doi.org/ 10.1001/jama.2019.21579 [published online ahead of print, 2019 Dec 17].

34. Armato SG 3rd, McLennan G, Bidaut $L$, et al. The lung image database consortium (LIDC) and image database resource initiative (IDRI): a completed reference database of lung nodules on CT scans. Med Phys. 2011;38(2):915-31. https://doi.org/10.1118/1.3528204 PMID: 21452728.

35. Armato SG 3rd, Meyer CR, Mcnitt-Gray MF, McLennan G, Reeves AP, Croft BY, Clarke LP, RIDER Research Group. The Reference Image Database to Evaluate Response to therapy in lung cancer (RIDER) project: a resource for the development of change-analysis software. Clin Pharmacol Ther. 2008; 84(4):448-56. https://doi.org/10.1038/clpt.2008.161 PMID: 18754000.

36. Clark KW, Gierada DS, Moore SM, et al. Creation of a CT Image Library for the Lung Screening Study of the National Lung Screening Trial. J Digit Imaging. 2007;20(1):23-31. https://doi.org/10.1007/s10278-006-0589-5 PMID: 1678359852.

37. Kinsinger LS, Anderson C, Kim J, et al. Implementation of lung cancer screening in the veterans health administration. JAMA Intern Med. 2017; 177(3):399-406. https://doi.org/10.1001/jamainternmed.2016.9022 PMID: 28135352.

38. Heuvelmans MA, Vonder M, Rook M, et al. Screening for early lung cancer, chronic obstructive pulmonary disease, and cardiovascular disease (the Big3) using low-dose chest computed tomography: current evidence and technical considerations. J Thorac Imaging. 2019;34(3):160-9. https://doi.org/ 10.1097/RTI.0000000000000379 PMID: 30550403.

39. Aldrich MC, Mercaldo SF, Sandler KL, et al. Evaluation of USPSTF lung cancer screening guidelines among African American adult smokers. JAMA Oncol. 2019;5(9):1318-24. https://doi.org/10.1001/jamaoncol.2019.1402 Published online June 27, 2019

40. Grubbs SS, Polite BN, Carney I Jr, et al. Eliminating racial disparities in colorectal cancer in the real world: It took a village. J Clin Oncol. 2013;31: 1928-30 201338. National Colorectal Cancer Roundtable: 80\% by 2018 Fact Sheet. Updated July 2014.http://nccrt.org/wp-content/uploads/80-by-2018FACT-SHEET.FINAL_.pdf39.

41. Jemal A, Siegel RL, Ma J, et al. Inequalities in premature death from colorectal cancer by state. J Clin Oncol. 2015;33(8):829-35. https://doi.org/ 10.1200/JCO.2014.58.7519 Epub 2014 Nov 10. PMID: 25385725.

42. Flores RM, Yankelevitz DF. Lung Cancer screening: the true benefit. Ann Thorac Surg. 2018;106(2):319-20. https://doi.org/10.1016/j.athoracsur.2018.03. 016 Epub 2018 Apr 12. PMID: 29654725.

43. Sevick-Muraca EM, Frank RA, Giger ML, Mulshine JL. Moonshot acceleration factor: medical imaging. Cancer Res. 2017;77(21):5717-20. https://doi.org/10. 1158/0008-5472.CAN-17-1698 Epub 2017 Oct 9.4 PMID: 28993413.

\section{Publisher's Note}

Springer Nature remains neutral with regard to jurisdictional claims in published maps and institutional affiliations.
Ready to submit your research? Choose BMC and benefit from:

- fast, convenient online submission

- thorough peer review by experienced researchers in your field

- rapid publication on acceptance

- support for research data, including large and complex data types

- gold Open Access which fosters wider collaboration and increased citations

- maximum visibility for your research: over $100 \mathrm{M}$ website views per year

At BMC, research is always in progress.

Learn more biomedcentral.com/submissions 